\title{
Nadia Metwally
}

\section{GLU்CK UND ELEND IM KAUFMANNSLEBEN}

\author{
Gattungstheoretische überlegungen \\ zu einem Thema des "bürgerlichen" Dramas \\ (Lillos 'Kaufmann' und Sternheims 'Snob')
}

Im 18. Jahrhundert erfolgte die Erhebung des Bürgertums zum Gegenstand tragischer Anschauung. Das "bürgerIiche Trauerspiel" war das dramatische Genre, worin im Zeitalter der Aufklärung - zuerst in England, dann in Deutschland - die Gestaltung des Tragischen in bürgerlicher Umwelt sich ausbildete. Dies galt als Gegenentwurf zu dem langlebigen Dogma der höfisch-klassizistischen Asthetik, das besagte, daß nur die Schicksale von Königen, Fürsten, Adligen Gegenstand der Trauerspiele sein dürften, während der Bürger als Lustspielfigur auftreten könne (Ständeklausel).

Die soziologisch orientierte Deutung verstand und versteht noch heute das Auftreten des Bürgers in der Tragödie, statt wie bisher als Gegenstand des Gelächters in der Komödie, als Außerung der selbstbewußten Mentalität des im 18. Jahrhundert wirtschaftich und gesellschaftlich erstarkenden Bürgertums.

In England war es George Lillo, der, gestützt auf ein starkes Bürgertum, 1731 den revolutionären schritt wagte, ein bürgerliches Trauerspiel ${ }^{2}$ "Der Kaufmann von London oder Begebenheiten Georg Barnwells" zu veröffentlichen. Es handelt sich um ein stück, das nicht

1 Diese Bezeichnung wird zwar noch nicht von Lillo selbst gebraucht, sondern erst in der französischen u̇bersetzung von Pierre Clément de Genève (1748) verwendet und von ihm durch Henning Adam von Bassewitz (1752) in die deutsche úbersetung übernommen. Zwar hat es auch andere úbersetzungen gegeben, aber der Erfolg und die Wirkung des "Kaufmann von London" waren in Deutschland durch Bassewitz vermittelt. In dieser übersetzung war Lillos Drama zwischen 1754 und etwa 1780 eines der meistgespielten Stücke auf den deutschen Bühnen. Auch wurde der Text in sehr kurzen Abständen immer wieder neuaufgelegt und nachgedruckt. (Bis 1781 sind mindestens 14 Ausgaben nachweisbar). Vgl. hierzu Daunicht, Richard: Die Entstehung des bürgerlichen Trauerspiels in Deutschland. Berlin 1963. S. $222 \mathrm{ff}$. 
nur in einem bürgerlichen, nämlich kaufmännischen Milieu spielt, sondern auch ein ökonomisch fundiertes bürgerliches Standesbewußtsein gegenüber der Aristrokratie bekundet.

Das bürgerliche Trauerspiel in England war also künstlerischer Ausdruck des Selbstbewußtseins einer fortgeschrittenen (Handels-) Bourgeoisie und fungierte zu deren ideologisch-moralischen stärkung.

Die Bedeutung von Lillos stück besteht darin, daß es den deutschen Dramatikern Mut machte, tragische Stoffe aus dem gegenwärtigen, alltäglichen Familienleben des Mittelstandes zu rührenden, gleichzeitig bürgerlich-häuslichen Tugenden empfehienden Dramen zu verarbeiten.z Die Gestaltung privater Sujets wird zur wesensbestimmenden Bedingung für das bürgerliche Trauerspiel. Den Spuren Lillos folgend, durchbrach Lessing die Ständeregel und machte den Bürger zum Tragödienhelden: 1755 führte er mit seiner "Miss Sara Sampson" das bürgerliche Trauerspiel in die deutsche Literatur ein. Damit realisierte er die Tendenz burgerlicher Aussonderung aus der feudalen welt und erhob den mitleidigen Menschen zum höchsten Tugendideal. Bürgerliche Personen sollten nach Lessing in erster Linie durch ihre Menschlichkeit rühren. Zweierlei hob er immer wieder am bürgerlichen Drama hervor: das Allgemeinmenschliche und das Rührende. Durch diese beiden Momente hebt sich das bürgerliche Trauerspiel von der heroischen Tragödie ab. Lessing überwand also die feudale Ständeklausel dadurch, daß er den Menschen abgelöst von seiner ständischen Gebundenheit zum Handelnden machen wollte. ${ }^{3}$

\footnotetext{
2 Unter dem Einfluß des englischen bürgerlichen Trauerspiels von Lillo wurde der Bürger selbst zum tragischen Helden. Damit erhob der neue Held für seine Klasse Anspruch aui die erhabensten Empiindungen, die bislang auf der Bühne dem hohen Adel vorbehalten blieben. ("Das kaufmännische Milieu des "Kaufmanns von London" findet man jedoch nur in Duschs "Der Bankerot" (1763) wieder, und auch dort kennzeichnenderweise ohne das unumwunden und leistungsstolz artikulierte bürgerliche Selbstbewußtsein des englischen Kaufmanns.) Guthke, Karl S.: Das deutsche bürgerliche Trauerspiel. Stuttgart 1976. S. $26 \mathrm{f}$.

3 "Die Namen von Fürsten und Helden können einem Stücke Pomp und Majestät geben; aber zur Rührung tragen sie nichts bei. Das Unglück derjenigen, deren Umstände den unsrigen am nächsten kommen, muß natürlicher Weise am tiefsten in unsere Seele dringen; und wenn wir mit Königen Mitleiden haben, so haben wir es mit ihnen als mit Menschen, und nicht als mit Königen." Lessing, G. Ephraim: Hamburgische Dramaturgie. Leipzig 1972. S. 105.
} 
Das bürgerliche Trauerspiel unterzog als Teil der Emanzipationsbewegung des Bürgertums im 18. Jahrhundert die bestehende Gesellschaftsordnung einer kritischen Analyse und setzte anstelle der ständischen Wertungen ethische werte der Tugend, Sittlichkeit, Würde etc., als deren Vertreter und Hüter es sich begriff.

Die Perspektiven des Trauerspiels sind bedingt durch die Infragestellung dieser Werte, deren Verlust im Selbstverständnis des Bürgertums eine existentielle Gefährdung bedeutete.

Dieser im Drama erscheinende Geist des Bürgertums wandelt sich entscheidend, bedingt durch geistes- und sozialgeschichtliche Faktcien, über die Jahrhunderte hin. Im Laufe der Entwicklung wird er sogar zum Gegenbild seiner selbst und erscheint entweder in positiver Selbstdarstellung oder als Gegenstand schärfster Kritik. Das "bürgerliche Trauerspiel" wird heute als eine "tote Gattung"s bezeichnet. War dieses einst ein Organ des bürgerlichen Emanzipationskampfes im 18. Jahrhundert, worin der Individualitätsanspruch der neuen Klasse und deren antifeudalen Opposition zum Ausdruck kam, so wird es im 20. Jahrhundert, bedingt durch geistes- und sozialgeschichtliche Faktoren, zur Zielscheibe des Spottes. Das Bürgertum wird anstatt Träger des Tragischen wieder Gegenstand des Witzes und des Komischen.

Gattungstheoretisch fundierte ubberlegungen zum "Kaufmannssujet" in Lillos Stück "Der Kaufmann von London oder Begebenheiten Georg Barnwells" (1731) und in Sternheims stück "Der Snob" (1914) können am Beispiel der Darstellung des Bürgers und dessen Moral eine gattungsgeschichtliche Bewegung aufzeigen.

Die folgende Untersuchung hat es sich deshalb zur Aufgabe gemacht, Lillos Drama als Prototyp des bürgerlichen Trauerspiels mit dem Lustspiel bzw. der Komödie ${ }^{5}$ "Der Snob" des Dramatikers Carl

4 Guthke, Karl S.: Das deutsche bürgerliche Trauerspiel. A.a.O. S. 1.

5 Czucka sieht, daB Beziehungen wie Schauspiel Drama und Lustspiel Komödie unter und gegeneinander nur schwer abzugrenzen seien. "Sie wurden deshalb heuristisch wie äqualente Begriffe behandelt, ohne daß damit unterstellt sei. es handle sich bei Abgrenzungsversuchen um einen bloßen Streit um Worte." Czucka, Eckehard: Komödie im 20. Jahrhundert. - Wedekind, Sternheim. Horvath und einige spätere. In: Komödiensprache. Beiträge zum deutschen Lustspiel zwischen dem 17. und dem 20. Jahrhundert. Mit einem Anhang zur Literaturdidaktik der Komödie. Hrsg. von Helmut Arntzen. Münster i984. S. 161. 
Sternheim aus seinem berühmten Dramenzyklus "Aus dem bürgerlichen Heldenleben" (1910-1920) zu vergleichen.

Die Angabe, ein bürgerliches Heldenleben benutzt eine Wendung, die ganz nur verständlich werden kann, wenn die darin liegende Negation des Begriffs "Bürgerliches Trauerspiel" bedacht wird. ${ }^{\circ}$

Mit dem Vergleich dieser beiden dramatischen Genres von Lillo aus dem 18. Jahrhundert und von Sternheim aus dem 20. Jahrhundert hofft dieser Aufsatz einen Beitrag zur Gattungsgeschichte des "bürgerlichen" Dramas zu leisten.

Das Drama Lillos will eine Moral lehren, die der ökonomischen Stärkung des Bürgertums im 18. Jahrhundert dient, indem sie das Selbstbewußtsein des Kaufmanns hebt und Müßiggang und Verschwendung verurteilt. Die explizite Thematisierung des Kaufmannsstandes als des Inbegriffs frühürgerlicher Berufspraxis macht das Drama gattungsgeschichtlich interessant. Lillo geht es darum, auf dem Umweg über die vorgegebene dramatische Handlung den Kaufmann und seine Standesideologie auf die Bühne zu bringen.

Für sein Trauerspiel wählt der englische Dramatiker einen Doppeltitel: "Der Kaufmann von London oder Begebenheiten Georg Barnwells". Einerseits handelt es sich um den Kaufmann Thorowgood durch seinen sprechenden Namen (Durchausgut) ethisch qualifiziert und andererseits um seinen Handlungsgehilfen Georg Barnwell, den Protagonisten der eigentlichen Tragödie.

In der Figur des redlichen Kaufmanns Thorowgood verkündet der Autor seine Hauptabsicht: Der Handel sei die Grundlage der menschlichen Gesellschaft, ihrer Kultur, ihres Glücks, und Führerin sei die Ordnung. Die Bedeutung des Kaufmannsstandes für das Ganze der Gesellschaft wird hervorgehoben. Schon in der ersten Szene werden die Kaufleute als Retter des Vaterlandes gepriesen:

Ihr werdet daraus abnehmen, wie viel so großmüthige Kaufleute, als wir, in gewissen Begebenheiten zur Rettung ihres Vaterlandes, und zur Versicherung desjenigen Glückes, welches sie demselben zu allen Zeiten verschaffen, beytragen können $[\ldots]^{7}$.

\footnotetext{
6 Czucka, Eckehard: Komödie im 20. Jahrhundert. A.a.O. S. 162.

? Lillo. George: Der Kaufmann von London oder Begebenheiten Georg Barnwells. Ein bürgerliches Trauerspiel. U̇bersetzt von Henning Adam von Bassewitz (1752). Kritische Ausgabe mit Materialien und einer Einführung.
} 
Lillos Standpunkt muß begriffen werden als Bestandteil einer bereits etablierten bürgerlichen Ordnung, die ihren Besitz verteidigen und sich eine günstige Entwicklung sichern will.

Die Konfrontation von Tugend und Laster bestimmt die Komposition des Trauerspiels: Tugendhaft ist ein Verhalten, das die handelsbürgerliche Ordnung stärkt; was sie schwächt, ist lasterhaft.

Von hier aus sind Fabel, Heldenwahl und Figurenkonstellation zu verstehen. Der unglückliche Held des Stückes, der junge Kaufmannsgehilfe Barnwell, ist zu der durch Thorowgood repräsentierte ordnung in Widerspruch geraten. Er unterliegt der Verführung einer Dirne, wird zlim Dieb und Mörder und muß für seine Verbrechen mit dem Leben bezahlen. Auch die Rechtspflege, die hier geübt wird, dient der Erziehung zu bürgerlicher Ordnung. Lillo nutzte das Tugend-Laster-Motiv zur Propagierung einer bereits befreiten und relativ konsolidierten großbürgerlichen Gesellschaft. ${ }^{*}$ Die Bindung des Sujets an den englischen Kaufmannsstand gab dem Tugendproblem doch eine soziale Fixierung, die in England in Anbetracht der politischen Bedeutung des Handelsbürgertums gesamtnationales Interesse weitgehend beanspruchen konnte. Der sensationelle Erfolg ging vor allem auf die rührende Wirkung des ungewohnten bürgerlichen Sujets zurück. "Der Kaufmann von London" stellt ein tragisches Geschehen im bürgerlichen Milieu dar. Die Neuartigkeit seines Vorgehens wurde Lillo von seinem ersten Herausgeber Davies (1775) bestätigt:

No writer had ventured to descend so low as to introduce the character of a merchant, or his clerk into a tragedy. ( $K$ 123)

Lillo erhebt den Anspruch, den Wirkungsbereich der Tragödie zu erweitern. Das geschieht durch die Veränderung ihres Gegenstandsbereichs. "Plays founded on moral tales in private life" ( $K$ 110) gewinnen ein breites Publikum für die Bühne. Die ästhetische Qualität macht er von der Quantität der Wirkungen direkt abhängig: je größer das Publikum ist und je intensiver es angesprochen wird, desto vollkommener ist das Kunstwerk. Da es also in erster Linie darauf an-

Hrsg. von Klaus-Detlev Müller. Tübingen 1981. S. 7. (Im folgenden zitiert mit der Sigle K).

s Lessing wollte aber durch die "rührende Verbindung" von Vollkommenheit und Unglücksfällen das Mitleiden seines Publikums erregen. 
kommt, dem Publikum deutlich zu machen, daß seine Probleme zur Diskussion stehen, wird der hohe Stand, der für die Tragödienhelden verlangt wird, (Ständeklausel) zu einem Hindernis für die Wirkungsmöglichkeiten, da die Leiden und Schwächen von Königen und Fürsten für die Masse der Zuschauer von keiner unmittelbaren Bedeutung sind. Die Abweichung von der ständischen Konvention hat eine erhebliche Erweiterung der Wirkungsmöglichkeiten zur Folge, da die Erfahrungen eines Handlungsgehilfen für ein großes Publikum sehr viel relevanter als die gekrönten Häupter sind.

Lillo argumentiert deshalb nicht im Namen des Bürgertums als einer neuen sozialen Schicht, sondern als Anwalt der Tragödie, die in Gefahr steht, ihrem Anspruch als höchster Gattung nicht zu genügen, wenn sie ihren Wirkungsbereich verengt. ( $\mathrm{K}$ 125)

Bemerkenswert ist deshalb sein Rückgriff auf eine alte volkstümliche Ballade, ${ }^{\circ}$ deren Gegenstand die Geschichte des unglücklichen Londoner Handlungsgehilfen war.

Ihre Wirkung ist erprobt: Sie hat schon unzählige Augen mit Tränen gefüllt und sich damit als Tragödiensto if qualifiziert. Zugleich kann sie als moralisches Exempel dienen. indem sie vor den Folgen der Lasterhaftigkeit warnt. ( $K$ 126)

Einerseits waren es also die sentimentalen Wirkungen des Balladenstoffes, andererseits die bürgerliche Selbstdarstellung des Kaufmanns, die den Erfolg des bürgerlichen Trauerspiels garantierten.

Der junge Handlungsgehilfe Georg Barnwell, der von der raffinierten und geldgierigen Buhlerin Millwood verführt wird, bezahlt das erotische Abenteuer zunächst mit dem Verlust seiner Unschuld und seines Seelenfriedens und dann, zum Diebstahi an seinem Herrn angestiftet, mit der Preisgabe seiner Tugend. Er ist nicht mehr Herr seiner selbst und schreckt auch vor dem Verwandtenmord nicht zurück. Im Gefängnis und angesichts eines schmachvollen Todes findet er zu sich selbst zurück und stirbt auf erbauliche weise.

\footnotetext{
- In seinem Prolog zum "London Merchant" nennt Lillo "a fam'd old song" als Quelle seines Trauerspiels: "A London' prentice ruin'd is our theme, Drawn from the fam'd old song that bears his name." (K 112). - Die Ballade handelt vom Verbrechen und Tod Georg Barnwells. Sie besteht aus zwei Teilen und kreist um das Motiv von Besitz und Geld. Sie wurde in Percys Reliques of Ancient English Poetry aufgenommen. Vgl. K 113-118.
} 
Obwohl das Private als Gegensatz zum Historisch-Politischen der herkömmlichen Tragödie hier Gegenstand des Dramas wird, verweisen noch einzelne Handlungselemente wie Mord und Hinrichtung auf die heroische Tragödie. Auch erhält die Handlung historische Tiefe dadurch, daß sie in der Regierungszeit Elisabeth I. spielt bzw. um 150 Jahre zurückverlegt wird. Trotz dieses Verlustes an Aktualität, aus der Lillo den Wirkungsbereich der Tragödie zu begründen versuchte, wurde die beabsichtigte Wirkung, Abschreckung und Warnung vor dem Laster zu erzeugen, erzielt. Im Gespräch zwischen Thorowgood und seinem zweiten Handlungsgehilfen Trueman (ebenfalls durch seinen sprechenden Namen "ehrlicher Mann" ethisch qualifiziert) wird der Bürger mit dem Adel gleichgestellt:

Der Kaufmannsstand ist den Edelleuten so wenig unanständig, so wenig er sie davon ausschliesset [...]. (K $7 \mathrm{f}$.)

Trueman weist darauf hin, daß die gemeinsame Politik von der Königin und den Kaufleuten dem Land Geld und Blut erspart. Das gute Einvernehmen zwischen der Königin und ihren Londoner Kaufleuten wird gepriesen. Die Glorifizierung Elisabeths I. liegt im Interesse des Bürgertums.

Große Königin! Wie wenig gleichest du denen Fürsten, die, unter dem Vorwand der gemeinen Gefahr, ihre Unterthanen durch unerträgliche Steuren- und Erpressungen unterdrücken. (K 8)

Das bürgerlich-kaufmännische Ethos wird nicht nur dem aristokratischen rangmäßig gleichgestellt, sondern erweist sich sogar als menschlicher, insofern es den Krieg als die dem Adel gemäße Form der Konfliktlösung überflüssig macht. Die Abhängigkeit der Politik von der ökonomie wird hier deutlich.

Die Expositionszene ist insofern von Bedeutung, als Barnwell mit seinem Verstoß gegen die Tugend nicht nur die individuelle Moral, sondern auch die Standesethik verletzt. Demzufolge wird das Laster, dem Barnwell verfällt, noch lasterhafter gemacht, die Tugend, verkörpert in seinem Freund, dem ehrlichen Handlungsgehilfen Trueman, tugendhafter und nachahmenswerter gezeigt.

Das bürgerliche Selbstbewußtsein, das Thorowgood so nachdrücklich ausspricht, wird auch für den privaten Bereich der Familie wirksam. 
Der Umgang mit "vornehmen Standespersonen" ist Pür den Kaufmann selbstverständlich. Maria, Thorowgoods Tochter, bestätigt ihrem Vater die Gleichwertigkeit von stand und Verdienst:

Eine Standesperson, welche mit einem Kaufmann von Ihrer Art Freundschaft hält, erzeiget demselben allerdings eine Ehre, jedoch erniedriget sie sich dadurch im geringsten nicht. (K 9)

Dies bewahrheitet sich auch, denn Adlige bewerben sich um Maria.

Symptomatisch für die soziale Situation des aufsteigenden Bürgertums ist es, daß Thorowgood als Vertreter des aufsteigenden Standes Wert darauf legt, seinen Rang durch die Verheiratung seiner Tochter mit einer vornehmen Standesperson zu erhöhen. Maria, durch ihre heimliche Liebe zu Barnwell befangen, läßt sich deshalb nicht durch die Ehre einer Standeserhöhung blenden:

Titel und Vorzüge, welche uns blos die Geburt giebet, sind nicht vermögend, mein Herz zu reitzen. (K 10)

Auch Thorowgood weiß die persönlichen Verdienste vor den Vorzügen der Geburt zu schätzen:

Freylich sind die Vorzüge der Geburt und der Glücksgüter nicht dasjenige, welches einem Menschen die Hochachtung der Leute zuwege bringen kann, wenn ihn nicht auch dabey seine persönlichen verdienste noch mehr über andere erheben: Doch bey dem, der seines Adels und seiner Reichthümer würdig ist, sind es allerdings wahrhafte und würkliche Vortheile, welche seine Tugenden in das helleste Licht setzen. ( $\mathrm{K} 10$ )

Er sieht zwar in der Verbindung von Tugend und Adel die vollkommenste Lebensform, übt aber auf seine Tochter keinen Zwang aus, weil er die Ehe als Liebesgemeinschaft versteht. Maria liebt Barnwell heimlich und ist auch als Opfer bereit, den Geliebten zu decken. Sie veranlaßt Trueman, Barnwells Unterschlagung aus ihrem Vermögen zu ersetzen, um ihm seinen "ehrlichen Namen", dessen Verlust für den Kaufmann unersetzbar ist, zu retten. Sie gehört zu den Empfindsamen und ist eher bereit, die "Martern einer heimlichen Liebe" ( $K$ 49) mit ins Grab zu nehmen, wern Leben, Ehre und Glückseligkeit des Geliebten nur um diesen Preis zu retten sind. Sie bemüht sich darum,

einen jungen Menschen der öffentlichen Schande zu entreißen, der sich zwar von dem Wege der Tugend verirret hat, aber 
auf demselbigen doch wieder zu rechte gebracht werden kann, ( $\left(\begin{array}{ll}\mathrm{K} & 39\end{array}\right)$

weil sein Verbrechen seiner Natur widerspricht.

Im Unterschied zu Barnwell, der das Opfer seiner von Millwood geweckten Sinnlichkeit ist, weiß Maria ihren Begierden zu widerstehen, da sie ihre Erfüllung nicht mit Reue und Gewissensqual erkaufen will. Jedoch empfindet sie den Verzicht als Martyrium:

Allein, was ist eine Stunde, ein Tag, ja ein Jahr voll Leiden

gegen eine ganze Lebenszeit voll Marter und Qual? (K 37)

Maria ist das Gegenbild der skrupellosen Verführerin Millwood, die in Barnwell nur ein Werkzeug sieht, um zu Geld zu gelangen. Die Würde und die objetive Bedeutung des Kaufmannsstandes machen Maria in ihrer Gattenwahl relativ unfrei: sie kennt die Verpflichtungen, die an die reiche Erbin gestellt werden. Erst kurz vor seiner Hinrichtung gesteht Maria Barnwell ihre Liebe. Entsagung und Verzicht sind vieImehr das Zeichen einer Tugend, die ihre Ansprüche nicht um jeden Preis durchsetzt und die soziale Bindungen respektiert.

Marias Gehorsam gegenüber dem Vater steht in Kontrast zu Barnwells Bereitschaft, auf Millwoods Verlangen hin sogar seinen geliebten Onkel zu ermorden. Auf solche Kontrastierungen sind Personengestaltung und Handlungsführung angelegt.

Leidensbereitschaft und Selbstaufgabe werden von den Tugendhaften als Preis für das Bewußtsein ihrer Vollkommenheit verlangt, die sie vor der Geiahr bewahren, in Schande zu geraten.

Während sich Maria als Märtyrerin fühlt, weil sie ihre Begierden beherrscht, wird Millwood als eine "eingefleischte Teuflin" bezeichnet, die sich die Mittel zu einem sinnenfreudigen Leben verschafft. Inr Werkzeug ist Barnwell. Geldgier ist ihr ausschließlicher Antrieb, das Motiv, das für sie alle sittlichen Normen außer Kraft setzt und das sie veranlaßt, ihre Schönheit und ihren Verstand als Verführerin zu mißbrauchen.

Millwood benutzt ihre auf Erfahrung begründeten Kenntnisse des männlichen Verhaltens dazu, ihre weiblichen Reize gezielt einzusetzen, um zu GeId zu kommen. Planvoll und allmählich wird der unerfahrene Barnwell um seine Ehre (Unschuld und Gewissenhaftigkeit) gebracht, bis er schließlich ganz orientierungslos wird und eine Umkehr nicht mehr möglich ist. Er ist aber unfähig, nach dem Mord an seinem on- 
kel auch noch den Raub auszuführen, was Millwoods Pläne durchkreuzt. Dies veranlaßt sie, die Mordtat zu verurteilen und sich somit auf den Boden der geltenden Moral zu stellen. Jedoch bleibt diese Moral aber in ihrer Forderung des Raubes unberücksichtigt.

Die guiltige Wertordnung wird also von der Verbrecherin nicht negiert, sondern lediglich für ihre Person und ihre Interessen suspendiert. ( $\mathrm{K}$ 136)

Schon bei ihrem ersten Auftritt (I, 3) sitzt Millwood am Schminktisch, um sich jene Maske zuzulegen, deren Opfer Barnwell werden wird. Sie bekennt sich ausdrücklich zum Prinzip der Täuschung:

Wenn es eine Würkung der Verstellungskunst bey dem Frauenzimmer ist, dafür angesehen $\mathrm{zu}$ werden, was sie nicht, um dasjenige, was sie würklich sind, desto beliebter zu machen, und allezeit grade das Gegentheil von dem zu reden, was sie denken, so ist gewiß bey mir nichts natürliches, sondern alles gekünstelt. ( $\left.\begin{array}{ll}K & 14\end{array}\right)$

Das erklärt die Verwirrung des naiven Kaufmannsgehilfen, der zeitweise zwischen gut und böse nicht mehr unterscheiden kann, weil er durch falschen schein verfuhrt wird (durch falsche Tränen, scheinbare Verwirrung und Scham, vorgetäuschtes Unglück, finglerte Tugend). Millwood bestätigt durch ihre Handlung folgende von ihr im voraus formulierte These:

Es ist eine von allen Menschen angenommene und eingeführte Grundregel: $D a ß$ ein Frauenzimmer ohne Tugend, so wie eine Mannsperson ohne Ehre, auch die niederträchtigste Handlung $\mathrm{zu}$ begeben vermögend ist. ( $\mathrm{K} 12$ )

So wie Millwood sich von vornherein als "ein Frauenzimmer ohne Tugend" einführt, so prophezeit Lucia, ihr Kammermädchen, daß Barnwell über die Stufen des Ungehorsams gegen seinen Herrn und eines "weiten Gewissens" "uns bald gleich werden", also dem Laster verfallen wird:

Ein Laster ist allezeit der Grund eines neuen. Nichts ist natürlicher, als dieses. ( $\mathrm{K}$ 34)

Lillo geht es in erster Linie darum, Abschreckung und Warnung zu erzeugen. Deshalb führt er die Iasterhafte Intrige nicht als spannende Handlung vor, sondern unterwirft sie moralisierender Kritik. Auch läßt er einen großen Teil von Millwoods Aktionen berichtend referieren und demzufolge Anlaß zu kritischer Reflexion geben. 
Millwoods Helfershelfer sind ihr Kammermädchen, Lucia, und ihr Diener, Blunt. Beide sind in ihre Pläne eingeweiht und springen in kritischen Situationen ein, wenn ihre Intrige zu scheitern droht. Das erinnert an die konventionelle Rolle der intriganten Diener in der Komödie. Bei Lillo ermöglichen sie ihrer Herrin unverstelltes Sprechen, was die Voraussetzung dafür ist, daß das Publikum ihre Maske von Anfang an durchschaut. Sie beurteilen nicht nur den Ablauf der Intrige, sondern auch ihren moralischen Gehalt, den Kampf zwischen Tugend und Laster. Lucia und Blunt fungieren also einerseits als Komplizen ihrer Herrin, andererseits aber auch als Kommentatoren, die die Handiungen der Millwood beurteilen. Sie zweifeln an ihren Verführungskünsten, schließen wetten über den Ausgang $a b$, staunen über die Geschicklichkeit Millwoods, mit der sie kritische Situationen meistert, gratulieren zum Erfolg.

Damit sind sie eine Art Publikum auf der Bühne - kritische und wertende Zuschauer der Vorgänge. ( $K$ 138)

Von einem bestimmten Punkt an erwacht das Mitleid der verdorbenen Diener für Barnwell, und sie erschrecken schließlich über Mordpläne: Mitleid und Furcht bewirken eine Katharsis bei den beiden, so daß sie nun gegen ihre Herrin aktiv werden, ohne jedoch das Schreckliche verhindern zu können. Ihre Distanzierung erfolgt als Gegenintrige und wechsel der Partei. Für Barnwell finden sie eine Entschuldigung, da er aus "übermäßiger Zärtlichkeit" handelt und in eine "entsetzliche Verblendung", geraten ist. Zum Gegenteil wird "die argIistige, die grausame Millwood" als eine "unersättiche Furie" verurteilt. Lucia bekennt:

Ihre Unmenschlichkeit macht, daß ich sie verabscheue. ( $\mathrm{K}$ 43)

In I, 3 und IV, 12 wird Millwoods Verhalten motiviert. Sie empfindet sich als Opfer der Männerwelt. Die männiche Sexualmoral sucht zwar das Vergnügen, den sinnichen Genuß, verachtet aber die Frauen, die es gewähren. Ihre Sinnichkeit hat Millwood zu einer leichten Beute für solche Männer werden Iassen. Da sie sich als Opfer empfindet, versucht sie sich als Entehrte an der Gesellschaft bzw. an den Männern zu rächen, die sie als Urheber ihres lasterhaften Verhaltens beschuldigt. Angesichts der gesellschaftichen Zwange bleibt ihr nichts ubrig, als mittels ihrer Sinnlichkeit den Unschuldigen zu ver- 
führen. Millwood ist nicht nur die Gegenspielerin Marias, sondern steht auch in Opposition zu Thorowgood. ${ }^{10}$

Während die beiden ersten Szenen des ersten Aktes der Moral des Kaufmannstandes gewidmet sind, bringen die darauf folgenden Szenen die entgegengesetzte Weltanschauung von Millwood.

Lillo gibt seinem stück einen erbaulichen Schluß, indem er den bußfertigen Sünder durch Reue zu sich selbst zurückfinden läßt und ihm so durch den Sühnetod in der Hinrichtung göttliche Vergebung verheißt. Er legt auch Barnwell folgende worte in den Mund, die er direkt an das Publikum richtet:

Auf diese Weise läßt die Gerechtigkeit des Himmels einen Unglücklichen umkommen, um durch ein so schreckliches Exempel ein ganzes Volk dem Verbrechen zu entreißen. ( $K$ 73)

Hier wird die Wirkungsintention des Autors unmittelbar formuliert, der damit versucht, eine Rechtfertigung der Gattung zu geben. Inhaltlich ist es die bürgerliche Standesmoral, gegen die Barnwell und Millwood verstoßen. Im Unterschied zu Thorowgood, Maria und Trueman, die die Ideale der Tugendhaftigkeit, Triebunterdrückung und Tüchtigkeit verkörpern und demzufolge tugendhaft wirken, werden Millwood und Barnwell als negatives Exempel demonstriert, da sie sich gegen die bürgerliche Norm vergangen haben.

Thorowgood, als Hauptvertreter der herrschenden Gesellschaftsmoral, klagt Millwood an (IV, 12), von ihrer Schönheit und Intelligenz einen falschen Gebrauch gemacht zu haben und stimmt völlig mit der als gültig gesetzten Norm überein, die die juristische Bestrafung der úbeltäter fordert.

Millwood, die sich aber als Opfer dieser Moral empfindet, entgegnet, daß sie durch die Schule der Männer gegangen ist, daß ihre Bosheit eine Folge gesellschaftlicher Erfahrungen ist, die sie nicht etwa, wie Thorowgood unterstellt, "mit den allerverrücktesten und boshaftesten Mannespersonen", sondern mit "deren von allem Alter und in allen ständen" gemacht hat.

10 Diese Personenkonstellation bei Lillo finden wir im deutschen bürgerlichen Trauerspiel wieder. Sowohl die empfindsame, tugendhafte Maria als auch die lasterhafte, aristokratisch gesinnte Millwood tauchen in Lessings Drama "Miß Sara Sampson" und besonders in Goethes "Götz von Berlichingen" auf. (Bei Lessing "Sara" und "Marwood", bei Goethe "Maria" und "Adelheid". 
Sie sind alle so boshaft und gottlos, als ihre Macht es ihnen zulasset. ( $\mathrm{K} \mathrm{60}$ )

Ihre Anklage gilt besonders den Richtern und Priestern als Hütern der Ordnung, deren wirkliches Verhalten sie als eine Schule der Unmoral bezeichnet.

[...] diese haben mich gelehret, dadurch ihren Schutz zu verdienen, daß ich meine Laster Unschuldigen aufzubürden suche. (K 60)

Da Barnwell den ungeweckten Trieb nicht zu unterdrücken vermag, sondern ihn sinnlich auslebt, erliegt er leicht der Versuchung. Thorowgood zeigt Verständnis für Barnwell:

Seine Verbrechen sind groß; aber die Versuchung war auch hart. Sein Fall müsse uns dazu dienen, daß wir aus demselben die Menschenliebe und Sanftmuth lernen, bey allen unsern Handlungen Vorsichtigkeit gebrauchen, und uns selbst nicht gar $\mathrm{zu}$ viel vertrauen. ( $\mathrm{K}$ 63)

Trueman und Maria bewähren sich in dem Versuch, die Fehler stillschweigend zu korrigieren, um die bürgerliche standesmoral vor Verletzung zu bewahren.12

Barnwells Tod ist die Folge des Durchbrechens eines herrschenden Sittenkodex. Die Folgen seiner Handlungsweise werden nicht an seiner Familie gezeigt, sondern sein Verbrechen wird als eine bürgerliche Schande für den ganzen Stand, insbesondere den Kaufmannstand behandelt. Darin äußert sich das starke neue standesbewußtsein des englischen Bürgertums. ${ }^{12}$

Zweifellos bleibt die Kritik Lillos im allgemeinen moralischen Bereich. Untadelige Vorbilder werden vorgeführt, denen der Zuschauer nacheifern soll, andererseits stellt er Bösewichte, die am Ende ihre strenge und gerechte strafe erhalten, als abschreckendes Beispiel hin. Die Katastrophe ist Grundlage, die hauptsächlichsten Anliegen des Dramatikers zu realisieren: die maximale Erregung der Mitleidsfähigkeit des Publikums und die Explizierung der gesellschaftichen Bedeutung der Tugend in der umfassendsten weise.

\footnotetext{
$11 \mathrm{Daß}$ der Motivkern des Freundschaftsbeweises in der bürgerlich gewordenen Literatur an Gewicht gewinnt, je mehr ein Freund in das Unbürgerliche sinkt, hatte sich hier an den Lehrlingen Trueman und Barnwell in Lillos "Kaufmann von London" gezeigt.
}

12 Vgl. Eloesser. Arthur: Das bürgerliche Drama. Berlin 1893. S. 23. 
Im Unterschied $\mathrm{zu}$ Lillo lehnt Sternheim eine Orientierung des Menschen an sittlichen Grundsätzen mit dem Ziel der Selbstüberwindung als Beschränkung der persönlichen Freiheit ab.

Er fordert vom Menschen die Verwirklichung seiner "eigenen Nuance":

Einmaliger unvergleichlicher Natur zu leben, riet jedem Lebendigen ich, damit keine Ziffer, doch Schwung zu ihrer Unabhängigkeit entschlossener Individuen die Gemeinschaft bedeute, mit dem aus der Nation und der Menschheit ein Ziel allein erreichbar ist. ${ }^{13}$

Die Etablierung einer Norm widerspricht für Sternheim der geforderten Emanzipation des Individuums. Konflikte zwischen Pflicht und Neigung, die Schuld-Sühne-Problematik, alles, was den Menschen im Kampf gegen Schicksal, moralische Gebote oder Umwelt in Abhängigkeiten stürzt, sind Sternheim verhaßt.

Während der Held im bürgerlichen Trauerspiel sich den moralischen und ethischen Normen fügt oder an ihnen scheitert, verwirklicht der Held bei Sternheim seine Freiheit, indem er keiner Norm gehorcht. Dies unterscheidet ihm auch von dem ideologischen Zwängen ausgesetzten Bürger, der Ideale und Verpflichtungen theoretisch anerkennt, in seinem Verhalten aber davon abweicht.

In Unterschied zu Kants kategorischem Imperativ, nach welchem "jeder so handeln müsse, daß sein wille zugleich als Prinzip allgemeiner Gesetzgebung gelten konne", ist Sternheims Idee der Freiheit

die Idee einer radikal gedachten individuellen Freiheit, die Emanzipation des Menschen von allen moralischen Bindungen, insbesondere die vollkommene Ablehnung allgemeiner Vernunftsgesetze. Freiheit bedeutet für Sternheim Herrschaft des Lebens. Als Grundlage der Freiheit wird die ursprüngliche Veranlagung des Menschen als einzig guiltige Gesetzmäßigkeit anerkannt. Frei ist der Mensch nur dann, wenn er sich in übereinstimmung mit seiner Natur befindet, wenn er seine natürlichen Kräfte zur alleinigen Richtschnur seines Lebens macht. ${ }^{14}$

Diese Maxime führt Christian Maske in der Komödie "Der Snob" auf der Bühne vor. Das Problem der Selbstverwirklichung im streben

13 Sternheim, Carl: Das gerettete Bürgertum. In: Carl Sternheim. Materialienbuch. Hrsg. v. Wolfgang Wendler. Darmstadt und Neuwied 1980. S. 16.

14 Fehr, Hans Otto: Der bürgerliche Held in den Komödien Carl Sternheims. Phil. Diss. Freiburg i.Br. 1968. S. 30. 
nach sozialem Aupstieg liegt der Handlung zugrunde. "Der Snob" ist ein Teil der Maske-Tetralogie, die das Kernstück des "bürgerlichen Heldenlebens" bildet. Es-ist die Welt des Wilhelminischen Bürgertums, wie es Karasek richtig bemerkt, ${ }^{15}$ die in allen Stucken des HeIdenlebens vorgeführt wird.

In seinem ersten bürgerlichen Lustspiel "Die Hose" (1911), das seinen Dramenzyklus eröffnet, hat Theobald Maske nur eine Sorge, wegen des Skandals, den seine Frau Luise erregte, als sie während der Vorbeifahrt des Königs ihre Hose verlor, jetzt nicht ebenfalls seine Stellung als Beamter zu verlieren. Aber der Vorfall hat durchaus unerwartete Folgen: Zwei Männer - Scarron und Mandelstam sind bei dem überraschenden Anblick in Leidenschaft zu Frau Maske entbrannt. Sie beziehen als Untermieter zwei freistehende Zimmer in der Maskeschen Wohnung und machen Frau Maske den Hof. Am Ende jedoch ist der Gatte der Gewinner, seine Frau bleibt ihm treu, und das Abenteuer bringt ihm Geld und sogar auf Umwegen noch eine Geliebte ein. So hat die Affäre mit der Hose für Maske nur die angenehmsten Folgen. Maske sieht sich zum erstenmal finanziell in die Lage versetzt, sich ein Kind leisten zu können.

Sein Sohn ist Christian Maske, der im Mittelpunkt der Komödie "Der Snob" steht. Er ist eine Neuausgabe dessen, was Theobald schon vollkommen war. Stolz bekennt sich der Vater zu seinem Sohn:

In dir ist alles Maskesche um ein paar Löcher weitergeschnallt. ${ }^{16}$

Das Drama konstituiert sich im wesentlichen aus, der Analyse der Eigenschaften und Verhaltensweisen Christian Maskes im Hinblick auf seine gesellschaftiche Position. Dieser ist im Zuge des allgemeinen Aufstieges und wirtschaftlichen wettbewerbes Aufsichtsrat einer bedeutenden Industriegesellschaft geworden. Er steht im Schnittpunkt zwischen seiner ärmlichen Herkunft und seiner glänzenden Zukunft. Vorgeführt wird, wie er sich aller Hemmnisse entledigt, die sich seiner Karriere in den Weg stellen könnten. Er ist entschlossen, sich von allen Bindungen zu befreien, die ihn kompromittieren könnten. Brutal

15 Karasek, Hellmuth: Carl Sternheim. Hannover 1965. S. 20.

16 Sternheim, Carl: Der Snob. Komödie. Darmstadt und Neuwied 1981. S. 69. (Im folgenden zitiert mit der Sigle S). 
entlohnt er seine Geliebte, Sybil Hull, die ihn in schweren Jahren ernährte und ihm die nötigen gesellschaftlichen Umgangsformen beibrachte. Er zahlt sie für die gemeinsam verbrachten vier Jahre mit fünfprozentiger Verzinsung aus und zieht damit einen dicken Schlußstrich unter eine nicht mehr standesgemäßige Liebe. Aup die gleiche Weise verfährt er mit seinen EItern. Er kauft sich von ihnen los, weil er fürchtet, ihr ärmliches Auftreten würde seinen Plänen schaden, und schickt sie in die Schweiz. Als es vornehm wird, auch in feineren Kreisen arme, aber ehrliche und gepflegte Eltern vorzuführen, weil es die eigentlichen Verdienste deutlich machen könnte, will er sie ebenso unbedenklich zurückholen. Zur wirtschaftlichen Macht, die er sich bereits erobert hat, erkämpft er sich jetzt den gesellschaftichen Glanz. Er bittet den Grafen Palen um die Hand seiner Tochter Marianne. Da Palens finanzielle Verhältnisse zerrüttet sind, einigt man sich auf die Verbindung. Die Art und weise des Zustandekommens der Eheschließung im Gespräch mit dem Grafen macht deutlich, daß es sich dabei nicht um eine Frage von Liebe und Zuneigung handelt, was sich auch im Fehien jeder Liebesszene ausdrückt, sondern daß es für Christian eine rein gesellschaftliche Angelegenheit ist im Hinblick auf die Beseitigung des adligen Standesvorurteils gegen ihn, während für den Grafen dessen finanzielle Notlage ausschiaggebend ist. Um die letzten Schranken zwischen der adigen Marianne Palen, die inzwischen seine Frau geworden ist, und ihm niederzureißen, erzählt Christian Maske die Affäre um die Hose seiner Mutter neu: sie sei Ausgang gewesen für ein Verhältnis zwischen seiner Mutter und einem französischen Vicomte, der im Bois de Boulogne Zeuge des Malheurs geworden. Christians Andeutungen lassen der Phantasie seiner Gattin freien Lauf, die nun annehmen muß, er sei Produkt dieser Vereinigung, also adiigen Blutes. Er verbietet ihr,

für unser ganzes Leben, jemals daran zu rühren, jemals jemanden, auch mich selbst, ahnen zu lassen, was du vermutest, was du meinst. Ich heiße Maske und basta. (S 82)

Es handelt sich in dieser Komödie um einen Mann, der aus kleinen Verhältnissen in die große Welt vorstößt, deren MitŁel durchschaut und sie übersteigert und rücksichtslos gegen diese welt einsetzt.

Der Snob, Christian Maske, ist Produkt und Ausdruck einer bestimmten gesellschaftlichen Situation. Der bürgerliche Aufstieg in eine Welt, die noch feudales Gepräge hat, ist in der 
Gestalt konzentriert. Die Mittel, die nach oben führen, sind schonungslos freigelegt. ${ }^{17}$

Christian begreift den Kampf um den sozialen Aufstieg als Kampf eines jeden gegen jeden.

Kämpfe ums Dasein. Die habe ich auch durchgemacht und dabei ganz anders als Myriaden Boden in mir aufgerissen; von Trieben geschnell, flog ich durch den Brei der Bequemen, weil ich wußte, jenseits fängt erst das Leben an. (S $47 \mathrm{f}$.

"Der Snob" repräsentiert den gewisseniosen Karrieretyp, der die Herrschaftsschicht unterwandert, um an ihrer Macht zu partizipieren. Er hat es auf Marianne, die Grafentochter, abgesehen, um durch eine Heirat seine gesellschaftliche stellung noch mehr $\mathrm{zu}$ festigen.

Sternheim legt den Widerspruch einer Epoche bloß, die sich innerlich längst eingestanden hat, daß Verdienste das sind, was man verdient, aber äußerlich an der Fiktion festhält, Macht und Ansehen könne nur in erhabenen Stammbäumen auf wachsen. ${ }^{18}$

Christians Machtanspruche konzentrieren sich auf eine umfassende Einflußnahme auf seine Umwelt. Selbst in der Hochzeitsnacht drängen sich seine eigentlichen Bewußtseinsinhalte in den Vordergrund:

Mit sechsunddreißig Jahren bin ich Generaldirektor unseres größten wirtschaftlichen Konzerns. Kontrolliere einen fünften Teil des Nationalvermögens. (S 73)

Der bürgerliche Behauptungswille weitet sich aus zum totalen Machtanspruch:

Ich habe Macht zu dem Erdenkbaren aus der Kraft meines Blutes. (S 75)

Fußend auf der Wirtschaftsform des Kapitalismus, die er selbst mit ins Leben gerufen hat, strebt der Bürger nach der Machtübernahme im staat.

Der politisch unmündige Bürger wird sich des eigenen ökonomischen Machtpotentials bewußt und macht sich auf, die feudale Vorherrschaft im politischen Bereich außer Kurs zu setzen. In Christian dokumentiert sich der imperiale Aspekt bürgerlicher Wirtschaftsgesinnung. ${ }^{10}$

17 Karasek, Hellmuth: Carl Sternheim. A.a.O. S. 35.

1. Ebd. S. 38 .

10 Freund, Winfried: Die Bürgerkomödien Carl Sternheims. München 1976. S. 20 . 
Die Kapitulation des Grafen Palen vor dem Kapital zeigt den Bürger auf dem Höhepunkt seiner Macht.

Offenbar hängen im 'bürgerlichen Bewußtsein der Wilhelminischen Ära Macht und Adel so eng zusammen, daß die Dokumentation der Macht nur durch Anpassung an die aristokratische Lebensweise erscheint. 20

Christian sagt:

Ein Ziel ward gekrönt. Zerknirschung des Feindes, Verbeugung vor dem Sieger. (S 71)

Die Absicht des Grafen Palen, seine Tochter mit dem reichen Bürger zu verbinden, zeigt an, daß er die Zeichen der Zeit verstanden hat.

Der Adel hat seine prekäre Lage erkannt und begreift die Verbindung mit dem kapitalstarken Bürgertum als letzte Rettung. Er flüchtet sich nicht in wirklichkeitsfremde Utopien, sondern macht sich die geänderten Systembedingungen zunutze. Wenn er auch seinen traditionellen Platz an den Schaltstellen der Macht dem Bürger überlassen mußte, so bleibt ihm durch Anpassung an die geltenden ökonomischen Bedingungen die Rolle des Mindermachtigen. Sein Adelspradikat setzt er dabei als begehrten Tauschwert ein, da es die neu errungene bürgerliche Machtposition nachtraglich in den Augen der Welt legitimiert.21

Der Graf macht eine weitere Einflußnahme auf die Gesellschaft vom Mitverfügungsrecht über Kapital abhängig:

Besitz, welcher Art er auch sei, wird ersessen. (S 38) Weiter gesteht er seiner Tochter angesichts ihrer bevorstehenden Heirat mit dem reichen Bürger:

Ich habe in meinem Leben Sonderliches zustande zu bringen nie versucht, war nur ein Adliger mit Bewußtsein angeborener Besonderheiten. Offenbart dieser Mann, es bedarf keiner Vorfahren, gewisse unschätzbare Güter zu besitzen, bin ich in meiner Bedeutung vor mir selbst geleugnet. (S 38)

Der verarmte Graf Palen begreift die Verbindung mit dem kapitalstarken Bürger als letzte Rettung. Der verschuldete Graf ist beeindruckt von der Iuxuriösen Lebensgestaltung des Bürgers und wittert eine Chance zur Wahrnehmung eigener Interessen.

20 Ebd.

21 Freund, Winfried: Die Bürgerkomödien Carl Sternheims. A.a.O. S. 35. 
In der Sternheimischen Komödie geht es nicht um die Konfrontation von Tugend und Laster wie in Lillos Trauerspiel, sondern "die Komödienkontroverse ist hier der zu industriellen Profitinteressen therabgekommene' Adel und die zu feudaler Lebenshaltung theraufgekommene' Bourgeoisie. ${ }^{12 z}$

Die HandIung der Komödie ist im Unterschied zum bürgerlichen Trauerspiel nicht ideenbezogen.

Die Realisierung einer Idee weicht bei Sternheim der Materialisierung des bürgerlichen Egoismus. ${ }^{23}$

Im "Snob" steht das zentrale Ich im Mittelpunkt. Der Bürger Christian Maske strebt in Sternheims Komödie, nachdem er zu Reichtum und Ansehen gelangt ist, nach der Heirat mit einer Grafentochter, um sich gräflichen Glanz zu fügen. Die angestrebte Heirat mit der Grafentochter weist deutlich darauf hin, wie sehr der reichgewordene Bürger seine neu errungene Machtposition mit adligen Attributen zu dekorieren bestrebt ist. Auch in Lillos Trauerspiel war Thorowgood ais Vertreter des aufsteigenden Bürgertums bestrebt, trotz seiner Schätzung der persönlichen Verdienste vor den Vorzügen der Geburt, seinen Rang durch die Verheiratung seiner Tochter Maria mit einer vornehmen Standesperson zu erhöhen.

Doch kommt es bei Lillo zu keiner tatsächlichen úberbrückung der Standesunterschiede, da Maria aus heimlicher Liebe zu Barnwell alle adligen Bewerber zurückweist.

Für Christian Maske aber spielt die Liebe keine Rolle. In welchem Maße er jede Gefühlsregung und menschliche Anteilnahme verneint, verdeutlicht seine Erwiderung auf Sybils Geständnis:

Ich liebe dich, Christian. Du bist der Fehler in der Rechnung meines Lebens. (S 13)

Sie lautet:

So verdienst du in Not und Elend $\mathrm{zu}$ sterben. Da nimm den Kuß umsonst.- (S 13)

Für Christian ist die Eheschließung mit der Grafentochter vielmehr das Erklimmen einer weiteren Sprosse auf der gesellschaftlichen Stufenleiter. Die Aufnahme in die adlige Gesellschaft gelingt ihm,

22 Rilla. Paul: Essays. Berlin 1955. S. 203.

23 Freund, Winiried: Die Bürgerkomödien Carl Sternheims. A.a.O. S. 67. 
nachdem er seine kleinbürgerliche Herkunft und Vergangenheit von sich abgestreift und sich den im aristokratischen Bereich gültigen Normen und Verhaltensweisen angepaßt hat. Diese Anpassung erreicht er, indem er die Haltung eines Snobs einnimmt.

Dieser Begriff ist die Abkürzung für das Iateinische "sine nobilitate". Im 18. Jahrhundert war diese Abkürzung an den englischen Universitäten für die Abgrenzung des gemeinen Bürgerlichen gegenüber den jungen Lords und Grafen gebräuchlich. ${ }^{24}$

Der Begriff "Snob" bezeichnet ganz allgemein "den Mann unbedeutender Herkunft, der trotzdem den glänzenden Schein, die Teilnahme an der Lebensart der Vornehmen sucht", indem er

nicht mehr nach Trieben, Bedürfnisse, Leidenschaften, sondern nur noch nach Zwecken, Absichten, Eitelkeiten handelt, da ihm niemals Wert oder Unwert einer Sache, sondern nur ihre modische Geltung interessiert [.... $]_{0}^{25}$

Fehr stellt mit Recht fest, daß Christian "in Wahrheit kein Snob ist, wohl aber, und das mit Vollkommenneit, die Rolle eines Snobs spielt.20

In welchem Maße sich Christian verstellt, um seine Natur und seine wahren Absichten zu verbergen, zeigt schon die Abfassung der Dankadresse auf die Einladung des Grafen. (I, 2) Die WahI des Papiers, das angestrengte Suchen nach der geeigneten Vokabel zeugen von Christians Kenntnis der in der Aristokratie vorherrschenden Lebensauffassung eines reinen Formalismus, dem er sich zu seinen Zielen anpaßt. Um der perfekten Formulierung willen wird aus der beabsichtigten Zusage schließlich eine Absage. (I, 5)

Bis hinein in den privaten Bereich dokumentiert sich dieses den adligen Lebensformen angepaßte Verhalten. Der Tod seiner Mutter wird inm zum Anlaß, seine Kleider zu wechseln; statt der Trauer kommt es zu deren Demonstration:

24 Vgl. Fehr, Hans Otto: Der bürgerliche Held in den Komödien Carl Sternheims. Phil. Diss. Freiburg i. Br. 1968. S. 101.

25 Bab, Julius: Snobismus. In: Die Gegenwart. Berlin. Jg. 43. 1914. Nr. 7. S. 103ff. Zitiert nach: Fehr, Hans Otto: Der bürgerliche Held. A.a.O. S. 101.

26 Fehr, Hans Otto: Der bürgerliche Held. A.a.O. S. 102. 
Ich werde mit dem Architekten, einem Bildhauer wegen des würdigen Grabmals gleich mich ins Vernehmen setzen. (S 52)

Sybil, eine der Frauengestalten bei Sternheim, ist Christians seitherige Geliebte, die nicht nur seine kaufmännischen Talente entdeckt und gefördert hat, ihr verdankt er den Beginn seiner Karriere, denn sie hat ihm Geschmack und Manieren jener Klasse beigebracht, in die er aufzusteigen gedenkt. Mit der Erfüllung dieser Aufgaben erlischt auch gleichzeitig ihre Bedeutung. Seine Dankbarkeit empfindet er als Schuld, die er mit einer Summe Geld aus der Welt schafft: moralischer Verpflichtungen entledigt er sich durch finanzielle Abfindung. Jede Gefühlsregung und jede menschliche Anteilnahme verneint er und handelt nur als Bourgeois. Er zahlt seiner Geliebten aus nach eiriem genau geführten Kontobuch, in dem er alle erfahrene Liebe und Förderung aufrechnet. Was für sybil "Lebenssache" ist (I, 1), erledigt Christian als ihr gelehriger Schüler, der sie mit ihren eigenen Waffen schlägt, wie ein Geschäft:

$\mathrm{Du}$ weißt, ich habe nach deinen Lehrsätzen recht. Nur schmerzt es, sie auf dich angewendet zu sehen. Ich trete in öffentliches Leben. Nirgends ein Fehler im Kalkul. (S 10)

Die Auseinandersetzung mit Sybil trägt den Charakter von Verhandlung; das Gespräch zwischen beiden zielt nicht auf Verständigung, sondern auf Abmachung, Sybil fügt sieh, sie läßt sich kaum anmerken, wie schwer es ihr fällt. Der Frau kommt hier nur eine episodische Bedeutung für den Mann zu. Ähnlich einer Ware wird sie nach Erlöschen ihres Konsumwertes belanglos.

Als Sybil nach ihrer endgültigen Verabschiebung auszudrücken versucht, "wie niedrig" sie Christian "empfinde", (S 50) droht jener kalt:

Entstellst du mich mit Verdächtigungen wie den eben geäußerten vor dir selbst, zerstörst du dir das Andenken deiner großen Leidenschaft. Doch bleibt das deine Sache. Wagst du sie vor anderen, drohen dir unnachsichtlich die Gerichte. (S 50)

Marianne ist die zweite Frauengestalt in Sternheims Komödie. Verarmt und verschuldet fuhrt Graf Palen sie in die Arme des Bürgers, weil er seine dekorativen Bedürfnisse durchschaut.

Marianne wird zum Gegenstand einer auschlieblich ökonomisch bestimmten Transaktion, in deren Verlauf es darum geht, den 
Gebrauchswert der adligen Tochter für den Bürger entsprechend herauszustellen. ${ }^{27}$

Marianne wehrt sich aber dagegen, nur als objekt zu fungieren. Auf die Frage des Grafen:

Nähmst du ihn auch, besäße er seine Reichtümer nicht, die uns aus einer Reihe schwieriger Umstände retten. (S 36)

erwidert sie:

Auf diese Frage kann ich nicht antworten. Als du ihn die ersten Male brachtest, wußte ich kaum, wer er war; nichts von seiner Situation. Mein Gefühl entschied frei. (S 36)

Sie gesteht weiter:

Von diesem Mann empiange ich die erste volle Empiindung meines Lebens. Noch schwärmt sie ungeklärt, und mit Glück ist Abwehr gemischt. Ein seliges Geheimnis, das sich natürlich entdecken, doch nicht führen lassen will. (S 38)

Hier zeigt sich, daß Marianne sich berelts gefühsmäßig zu Christian hingezogen gefühlt hat, ohne etwas von seinem Reichtum gewußt zu haben, was besagt, daß für sie eine Verbindung mit Christian nicht nur eine reine Vernunftehe bedeutet. Gleichzeitig jedoch verbindet sich für sie Glück mit Reichtum. Sie findet es schön und angenehm "[...] mit solchen Dingen leben zu dürfen". (S 36)

Um Marianne nach der Heirat "zu grenzenloser Ehrfurcht" zu bringen, bedient sich Christian noch eines stärkeren Mittels, des stärksten von allen. Es handelt sich um die Umänderung seiner Herkunft. Es beginnt bei den Vätern. Wir hören, daß schon der Großvater 'ein tolles Huhn' (S 75) war. Der Vater Theobald aber 'ist eine Persönlichkeit'm.

Mutter Luise kam "aus einem Jahrhunderte alten Bauerngeschlecht." (S 77) Das Letzte, worauf alles hinausgeht, ist die Statuierung einer höheren Herkunft, und sei es selbst eine illegitime. Jene uns vom ersten Lustspiel her bekannte Episode von dem Fallen der Hose Mutter Luisens wird nach Paris in den Bois de Boulogne verlegt. Jene Episode habe Renoir beobachtet und ebenso ein französischer Vicomte. Renoir malt das Bild, und der Vicomte wird der erlauchte Vater Christian Maskes. Interessant ist, wie Christian das Gespräch so wendet, daß Marianne selbst auf das Geheimnis kommen muß, das er,

$\overline{27}$ Freund, Winfried: Die Burgerkomödien Carl Sternheims. A.a.O. S. 49. 
den Diskreten spielend, nicht aussprechen $\mathrm{zu}$ wollen fingiert. Der Snob steht am Ende, nachdem er die Ehre seiner Mutter preisgegeben hat, mit der Pose des Siegers vor der in Bewunderung versetzten jungen Frau.

Statt Liebe sucht er Hochachtung, nicht als Geliebter und Ehemann, sondern als "der geniale Mensch" (S. 74) will er anerkannt werden. SowohI seine Frau als auch die Gesellschaft werden durch die Vorspielungen getäuscht.

Die Darstellung der Lüge als Entstellung der Wahrheit konstituiert das Ganze als bloßen Schein. Der Gewinn der höchsten Stufe der Individualität bedeutet zugleich ihren vollständigen Verlust, und sie ist so der Erweis der Nichtunterscheidbarkeit von der Masse. ${ }^{28}$

Während bei Lillo der Held in den tragischen Konflikt mit seiner Umwelt gerät und zum Schluß scheitert, da er gegen die sittliche Norm verstößt, setzt sich der Sternheimische Held mit Erfolg durch, weil er sich den gesellschaftlichen Normen und Verhaltensweisen durch die Verstellung, durch die Maske angepaßt hat.

Im Gegensatz zum traditionellen Helden, der im Einsatz für eine sittliche Idee in den tragischen Konflikt mit seiner Umwelt gerät, vermeidet der Sternheimische Held von vornherein jeden offenen Zusammenstoß mit der Gesellschaft durch das Prinzip der "Maske". Es gibt für ihn keinen ausweglosen Zwiespalt, keinen Widerstreit zwischen dem Anspruch des Ich und der Gesellschaft. ${ }^{20}$

Tugend und Laster spielen bei Sternheim keine Rolle mehr. Den Sieg des Guten und die Bestrafung des Bösen auf dem Theater Iehnt Sternheim dagegen $a b$, weil er darin eine Abweichung von den tatsächlichen Verhältnissen und eine ideologische Verfälschung der Wirklichkeit sieht. Er distanziert sich von den Dichtern, die diese "Bilderbuchtugenden ${ }^{\text {mao }}$ besingen.

Während sich Millwood in Lillos Drama nur zeitweise der Maske bedient, um die Männer zu verführen und sie zum objekt ihrer Wünsche zu degradieren, ist die "Maske" schon im Namen des Helden bei

28 Czucka, Eckehard: Idiom der Entstellung. Auffaltung des Satirischen in Carl Sternheims "Aus dem bürgerlichen Heldenleben". Münster 1982. S. 219.

20 Fehr, Hans Otto: Der bürgerliche Held. A.a.O. S. 116.

so Vgl. Sternheim, Carl: Das gerettete Burgertum. In: Wendler, Wolfgang: Carl Sternheim. Materialienbuch. A.a.O. S. 16. 
Sternheim fixiert. Hinter der Maske verborgen ist aber keineswegs mehr ein eigentliches, ein wahres Wesen. Die Maskierung ist im 'bürgerlichen Heldenleben' zu einem Selbstzweck geworden, wie die satirische Darstellung Sternheims zeigt, der damit eine Auffassung verschärft, die schon bei Nietzsche in Ansätzen formuliert zu sein scheint:

Der Intellekt als Mittel zur Erhaltung des Individuums entfaltet seine Hauptkräfte in der Verstellung; denn diese ist das Mittel, durch das die schwächeren, weniger robusten Individuen, sich erhalten, als welchen einen Kampf um die Existenz mit Hörnern oder schariem Raubtier-Gebiß zu führen versagt ist. Im Menschen kommt diese Verstellungskunst aui ihren Gipfel: hier ist die Täuschung, das Schmeicheln, Lügen und Trügen, das Hinterdem-Rücken-Reden, das Repräsentieren, das im erborgten Glanze leben, das Maskiertsein, die verhülende Konvention, das Bühnenspiel vor anderen und vor sich selbst, kurz das fortwährende Herumflattern um die eine Flamme Eitelkeit so sehr die Regel und das Gesetz, daß fast nichts unbegreiflicher ist, als wie unter den Menschen ein ehrlicher und reiner Trieb zur Wahrheit aufkommen kommen konnte. ${ }^{31}$

Im selben Moment aber, wo sich Christian Maske der Gesellschaft anpaßt, verliert er seine Identität, sein wahres "Ich".

Im "Snob" legt Sternheim die Fäulnisherde einer scheinbar blühenden, friedlichen, kraftstrotzenden Gesellschaft bloß. Seine Kritik gilt der bürgerlichen Gesellschaft der Wilhelminischen Zeit, dem "juste milieu", wie es sich selbst nennt, das er als lediglich von Geld und Machtgier beherrscht und alles Tun und Fühlen, auch Liebe und Ehe, danach ausrichtend darstellt.

Es ist die Kehrseite des deutschen Aufstiegs in den Jahrzehnten vor 1914, die hier aufs Korn genommen wird. ${ }^{32}$

Indem Sternheim den "Snob" zur Titelfigur erhebt, parodiert er den herkömmlichen Heldenbegriff. Christian verharrt in völliger Rücksichtslosigkeit und Gefühllosigkeit gegenüber seinen Partnern, die er zu bloßen Objekten und Instrumenten seines Machtstrebens degradiert. Während sich das tragische Ende des Helden bei Lillo also aus desser. Verstoß gegen die bürgerliche Moral ergibt, Peiert der Held bei Sternheim durch die Anpassung an die Gesellschaft seinen Triumph.

31 Nietzsche. Friedrich: ưber Wahrheit und Lüge im außermoralischen Sinn. In: Philosophisches Lesebuch 3. Hrsg. von Hans-Georg Gadamer. Frankfurt/M. 1970. S. 190 .

32 Rilla, Paul: Essays. A.a.O. S. 203. 